\title{
Análise teórico-experimental do módulo dinâmico de compósitos asfálticos com argila calcinada
}

\section{(Theoretical-experimental analysis of dynamic modulus of asphalt mix with calcined clay)}

\author{
A.C. L. da Silva, C.A.da Frota \\ Grupo de Geotecnia, Faculdade de Tecnologia, Universidade Federal do Amazonas, \\ Av. Gen. Rodrigo Octávio Jordão Ramos, 3000, Manaus, AM 69077-000 \\ cleiton.acls@hotmail.com,cafrota@ufam.edu.br
}

\begin{abstract}
Resumo
$\mathrm{Na}$ análise mecanicista das misturas asfálticas, o módulo complexo é um parâmetro de importância fundamental para formulação de modelos constitutivos de sua estrutura, pois retrata tanto a parcela do comportamento elástico, decorrente dos agregados, quanto o comportamento viscoso, advindo do ligante asfáltico. A primeira é representada pelo módulo dinâmico e a segunda pelo ângulo de fase. Ambos os parâmetros dependem da temperatura e da frequência de carregamento. Como não é possível medi-los para toda temperatura e carregamento, obtêm-se funções que representem esses parâmetros, utilizando-se tanto ajustes genéricos como modelos de previsão do comportamento. Analisou-se a viabilidade de diversas modelagens referente ao módulo dinâmico de compósitos asfálticos com argila calcinada, obtidos mediante ensaios de flexão a quatro pontos. Observou-se que podem ser utilizados com eficiência tanto regressões múltiplas não lineares quanto as curvas mestras, com translação entre frequência e temperatura. Equações fenomenológicas também podem ser empregadas com precisão, por meio das quais é possível extrapolar os dados, obtendo-se o valor de equilíbrio do módulo dinâmico quando a frequência tende ao infinito.
\end{abstract}

Palavras-chave: mistura asfáltica, módulo dinâmico, argila calcinada, agregado sinterizado de argila calcinada, modelagem matemática.

\begin{abstract}
In the mechanistic analysis of asphalt mixtures, the complex modulus is a fundamental parameter for the formulation of constitutive models of its structure, since it represents the portion of elastic behavior, typical of the aggregate of the mixture, as well the viscous behavior, due to the asphaltic binder. The first is represented by the dynamic modulus and the second by the phase angle. Both parameters depend on the temperature and the loading rate. As it is not possible to measure them for all temperature and loading, functions that represent these parameters are obtained, using both generic fitting as predictive models. Various models for the dynamic modulus of asphalt composites with calcined clay, obtained by four-point bending tests, were analyzed. It is observed that both method, multiple nonlinear regressions as well mastercurves, can be used efficiently, with translation of frequency and temperature. Phenomenological equations can also be used accurately, by which it is possible to extrapolate the data, yielding the equilibrium value of the dynamic modulus when the frequency tends to infinity.
\end{abstract}

Keywords: asphalt mix, dynamic modulus, calcined clay, mathematical modeling, numerical modeling.

\section{INTRODUÇÃO}

O agregado sinterizado de argila calcinada (ASAC) apresenta-se promissor para localidades que apresentam dificuldades na obtenção de materiais pétreos, com vistas à produção de agregado graúdo (brita), e ao mesmo tempo possuem abundância de solos argilosos. Esta é a realidade presente em grandes regiões do Brasil, com destaque na pavimentação asfáltica de rodovias em trechos distantes dos centros urbanos, como são os casos da BR 163 (Santarém, PA - Cuiabá, MT) e BR 319 (Manaus, AM - Porto Velho, $\mathrm{RO}$ ), situações em que os agregados de argila calcinada se mostram viáveis técnica e economicamente [1-3].
Com uso desse mesmo material alternativo, o modulo dinâmico foi determinado anteriormente mediante ensaios de tração/compressão axial [4]. Desta feita, com o objetivo de agregar conhecimento ao comportamento desse agregado (ASAC) em composições asfálticas, realizaram-se estudos pioneiros com a determinação do módulo complexo desse material mediante ensaios de rigidez por flexão a quatro pontos. Também se inova com o método de dosagem das misturas, qual seja, a metodologia Superpave. Além da rigidez (módulo dinâmico), o teste para a determinação do módulo complexo também fornece o ângulo de fase, representativo do comportamento viscoso do material. Executou-se o experimento de forma dinâmica, mediante 
aplicação de pulsos de carga senoidais com o monitoramento dos deslocamentos, também senoidais. Esse ensaio é denominado como módulo de rigidez ou módulo complexo $[5,6]$. O módulo complexo varia em função da temperatura e da frequência de aplicação da carga, representativa da velocidade dos veículos sobre o pavimento. Como os ensaios são limitados a valores pontuais pré-estabelecidos, evidenciase a importância da adoção de modelos matemáticos que possam predizer o comportamento em circunstâncias diversas daquelas situações preconcebidas, ou seja, para temperaturas e frequências distintas das normalmente adotadas nos experimentos laboratoriais. Diante do exposto, o presente trabalho objetiva o estabelecimento de modelos matemáticos que possam ser utilizados para modelagem do módulo complexo de misturas asfálticas com argila calcinada (ASAC), com vistas à obtenção desse parâmetro mecânico para situações além das condições pontuais realizadas nos ensaios rotineiros.

\section{MATERIAIS E MÉTODOS}

Em substituição à brita na condição de material graúdo, utilizou-se o mesmo agregado sinterizado de argila calcinada (ASAC) anteriormente empregado para obtenção do módulo dinâmico de misturas asfálticas mediante ensaios de tração/ compressão axial [4], trabalho no qual podem ser encontrados os detalhes de suas análises químicas e físicas. Destaca-se que os índices de consistência indicaram LL (limite de liquidez $)=48 \%$ e LP (limite de plasticidade $)=22 \%$ (índice de plasticidade, IP $=26 \%$ ) e que a caracterização das amostras no estado natural e calcinada (ASAC) apontaram que a temperatura de queima não altera sua composição química (ASAC: $46,7 \% \mathrm{SiO}_{2}, 50,1 \% \mathrm{Al}_{2} \mathrm{O}_{3}, 1,5 \% \mathrm{Fe}_{2} \mathrm{O}_{3}, 1,1 \% \mathrm{~K}_{2} \mathrm{O}$, $0,6 \% \mathrm{TiO}_{2}$ ) [4]. Como agregado miúdo, empregou-se areia adquirida no mercado da construção civil local e, como ligante, o cimento asfáltico de petróleo CAP 50-70 fornecido pela Refinaria Isaac Sabbá (UN-Reman). Caracterizou-se o primeiro pela granulometria [7], densidades (Gsa e Gsb) e absorção [8] e, seguindo a AASHTO T-19/T 19M-93 [9], calcularam-se as massas específicas compactadas. O segundo foi analisado de acordo com as recomendações da Agência Nacional de Petróleo e Biocombustíveis (ANP). Os resultados são apresentados em [4]. Na preparação do compósito asfáltico utilizou-se como fíler mineral o cimento Portland, examinado pela sua massa específica real (DNER-ME 085/94) e granulometria (DNER EM 367/1997) e seguiu-se a metodologia Superpave na determinação da dosagem [10, 11], do teor de projeto, e na compactação por amassamento com uso do compactador giratório Superpave (CGS).

\section{Módulo de rigidez ou módulo complexo}

Dentro da análise mecanicista, o módulo complexo mostra-se de fundamental importância nos modelos constitutivos que buscam descrever o comportamento do concreto asfáltico. Esse parâmetro encontra-se inserido desde as análises iniciais até as avaliações de desempenho, que visam prever o desempenho e a vida útil do pavimento. Nos materiais elásticos, cujas solicitações sejam estáticas, a relação entre tensão e deformação específica recuperável denomina-se módulo de elasticidade. Nas misturas asfálticas, diante de seu comportamento viscoelástico, as deformações decorrentes da aplicação de uma carga são divididas em duas parcelas, uma consequente da resposta elástica e outra oriunda da participação viscosa. A primeira se manifesta de forma praticamente instantânea e a segunda é função do tempo de solicitação. Deste modo, uma forma de se medir a resposta elástica nos compósitos asfálticos é aplicar uma carga instantânea e medir a deformação correspondente, ou aplicar uma deformação instantânea e determinar a carga relacionada. Definiram-se, assim, os ensaios conhecidos como creep estático (axial) e módulo de relaxação, respectivamente.

No experimento denominado creep estático se encontra a função fluência resultante da aplicação de um carregamento instantâneo e constante ao longo do tempo, com o monitoramento dos deslocamentos. A citada função (creep compliance $), \mathrm{D}(\mathrm{t})$, pode ser calculada a partir da Equação A:

$$
D(t)=\frac{\varepsilon(t)}{\sigma}
$$

onde, $\varepsilon(\mathrm{t})$ é a deformação registrada ao longo do tempo e $\sigma$ é a tensão aplicada. O teste de módulo de relaxação é executado para a determinação da função $\mathrm{E}(\mathrm{t})$. Aplica-se um deslocamento instantâneo e constante ao longo do tempo, sendo acompanhada a força necessária para manter este deslocamento. Portanto, o módulo de relaxação pode ser calculado segundo a Equação B:

$$
\mathrm{E}(\mathrm{t})=\frac{\sigma(\mathrm{t})}{\varepsilon}
$$

Tais experimentos envolvem complicações relacionadas à aplicação de solicitações instantâneas, que na prática não pode ocorrer. Alternativamente, concebeu-se a aplicação de pulsos de carga senoidais com o monitoramento dos deslocamentos, tendo-se chamado módulo de rigidez ou módulo complexo, em que se aplicam pulsos de carga senoidais, induzindo-se deslocamentos no corpo de prova também senoidais, conforme as Equações C e D [5]:

$$
\begin{aligned}
& \sigma(t)=\sigma_{0} e^{i \omega t} \\
& \varepsilon(t)=\varepsilon_{0} e^{i(\omega t-\phi)}
\end{aligned}
$$

em que $\sigma_{0}$ e $\varepsilon_{0}$ são, respectivamente, as amplitudes de tensão e deformação, $\omega$ a frequência de aplicação de carga e $\varphi$ o ângulo de fase, sendo este a diferença de tempo entre os picos de tensão e deformação (Fig. 1).

A relação entre a tensão senoidal e a deformação define o módulo complexo (Equação E):

$$
\mathrm{E}^{*}=\frac{\sigma(\mathrm{t})}{\varepsilon(\mathrm{t})}=\frac{\sigma_{0}}{\varepsilon_{0}} \mathrm{e}^{\mathrm{i} \varphi}=\frac{\sigma_{0}}{\varepsilon_{0}}[\cos \varphi+\mathrm{i} \cdot \operatorname{sen} \varphi]=\mathrm{E}^{\prime}+\mathrm{i} \mathrm{E}^{\prime \prime}
$$




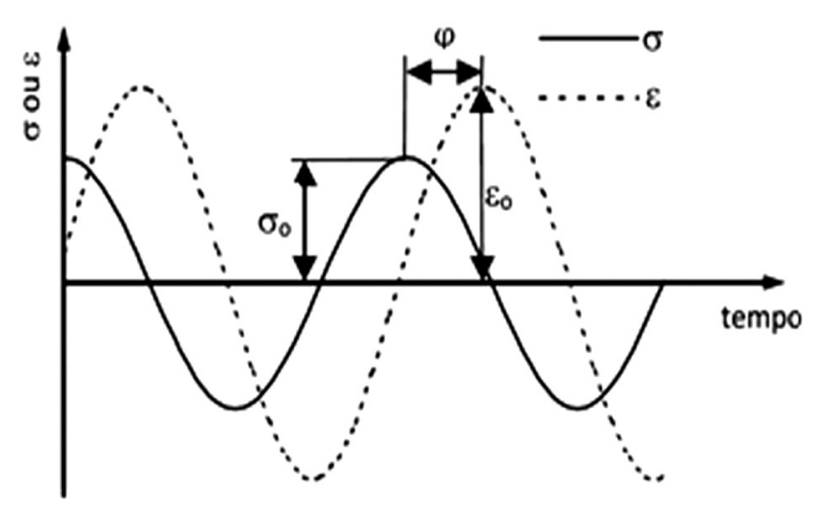

Figura 1: Esquema de solicitação e resposta no ensaio de módulo complexo.

[Figure 1: Diagram of loading and response in the complex modulus testing.]

onde, E' e E" representam, respectivamente, as partes real e imaginária do módulo complexo, que são conhecidas como módulo elástico (storage modulus) e módulo viscoso (loss modulus). O quociente entre a amplitude da tensão pela amplitude da deformação (Equação F) é definido como módulo dinâmico $\left|\mathrm{E}^{*}\right|$ e indica o valor absoluto do módulo complexo $\mathrm{E}^{*}$, podendo ser representado graficamente em um plano complexo (Fig. 2), dado por:

$$
\left|\mathrm{E}^{*}\right|=\frac{\sigma_{0}}{\varepsilon_{0}}=\left|\sqrt{\left(\mathrm{E}^{\prime}\right)^{2}+\left(\mathrm{E}^{\prime \prime}\right)^{2}}\right|
$$

em que $\sigma_{0}$ expressa a tensão dinâmica máxima e $\varepsilon_{0}$ a deformação axial recuperável máxima. Partindo-se do módulo dinâmico e o ângulo de fase, pode-se obter o storage (Equação G) e o loss modulus (Equação H):

$$
\begin{aligned}
& \mathrm{E}=\left|\mathrm{E}^{*}\right| \cos \varphi \\
& \mathrm{E}^{*}=\left|\mathrm{E}^{*}\right| \operatorname{sen} \varphi
\end{aligned}
$$

onde, E' (parcela real do módulo complexo) indica a parte elástica do material, E" (parcela imaginária do módulo) refere-se à parcela viscosa do material e o ângulo de fase $\varphi$ indica o ângulo de atraso de $\varepsilon_{0}$ relativo a $\sigma_{0}[12]$, sendo expresso por:

$$
\varphi=\frac{\mathrm{t}_{\mathrm{i}}}{\mathrm{t}_{\mathrm{p}}} \times 360
$$

onde, $t_{i}$ é a diferença de tempo entre os picos de tensão e deformação e $t_{p}$ o tempo de um ciclo de carga. Esse parâmetro permite conhecer as propriedades viscosas do material analisado. Deste modo, para um material puramente elástico $\varphi=0^{\circ}$ e para um material puramente viscoso $\varphi=90^{\circ}$.

O módulo dinâmico e o ângulo de fase variam com a frequência de aplicação de carga e a temperatura. No caso dos pavimentos asfálticos, a frequência está relacionada à velocidade dos veículos e a temperatura depende das

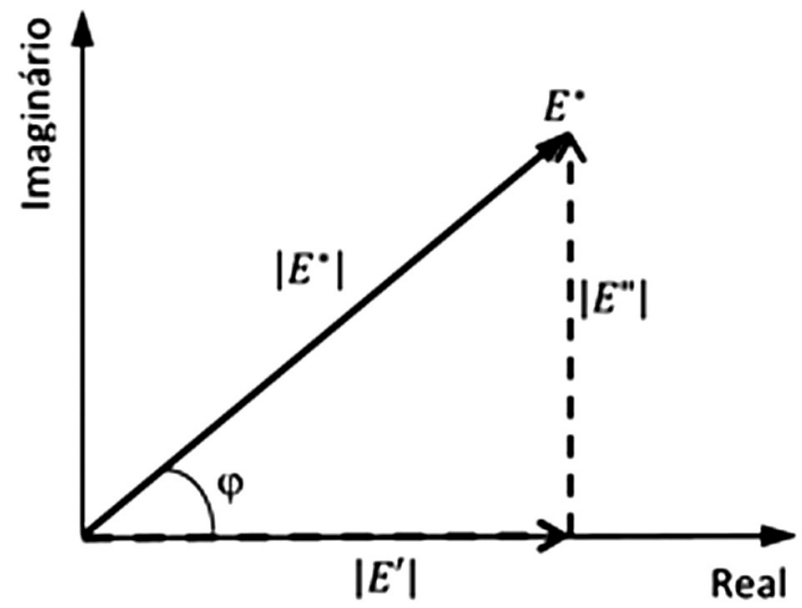

Figura 2: Representação gráfica do módulo complexo.

[Figure 2: Graphical representation of the complex modulus.]

condições ambientais. Portanto, os ensaios devem ser realizados variando-se essas condições para melhor representar os estados reais dos pavimentos. Como os ensaios laboratoriais consistem em se aplicar ao corpo de prova cargas repetidas, registrando-se os deslocamentos ao longo do tempo, devem ser estipulados, além da geometria do corpo de prova, frequência e modo de aplicação da carga. No presente trabalho, a determinação do módulo de rigidez se deu a partir da deformação de vigas mediante ensaios de flexão em quatro pontos [13].

Curvas mestras: diante das características dos compósitos asfálticos, há uma correlação de seu desempenho frente a frequências e temperatura. Nesse caso, o comportamento a baixas temperaturas e frequências deve ser o mesmo quando submetido a altas temperaturas e frequências. Pelo princípio da superposição, podem-se transladar curvas de uma determinada temperatura e frequência para outra temperatura, ajustando-se a frequência relacionada. O módulo dinâmico, então, constitui-se função apenas da frequência reduzida que pode ser obtida de acordo com as Equações J e K:

$$
\begin{aligned}
& \log \tilde{\omega}=\log \left(\omega \mathrm{a}_{\mathrm{T}}\right) \\
& \mathrm{a}_{\mathrm{T}}=\frac{\omega \text { Tref }}{\omega}
\end{aligned}
$$

onde, $\omega_{\text {Tref }}$ e $\omega$ são, respectivamente, a frequência na temperatura de referência e a frequência na temperatura em questão, e o parâmetro $\mathrm{a}_{\mathrm{T}}$ é o fator de translação horizontal (horizontal shift factor) [14].

Modelagem do módulo dinâmico: o pioneiro estudo na busca de modelagem fenomenológica do asfalto ocorreu em 1950, com Van der Poel, seguido por Jongepier e Kuilman no final da década de 60, e Dickinson e Witt nos anos 70 [15]. Apesar de algumas inconsistências, todos os trabalhos apontaram para a necessidade de se relacionar o comportamento reológico com o tempo de carregamento e a temperatura. Mais recentemente, durante o desenvolvimento 
do programa SHRP, Christensen e Anderson [16] propuseram a utilização de uma função derivada da distribuição de Weibull para representar a reologia do asfalto. Após esse trabalho, Marasteanu e Anderson [17] ofereceram uma modificação ao modelo original de Christensen-Anderson, introduzindo o chamado modelo CAM. Este foi usado em muitos estudos, sendo considerado um modelo fenomenológico eficaz para ligantes asfálticos não modificados, cujas propriedades estão dentro da faixa viscoelástica linear. No presente trabalho aplica-se o modelo proposto por Zeng et al. [18] para a formulação da curva mestra relativa ao módulo complexo (Equação L), considerada universal:

$$
\left|\mathrm{E}^{*}\right|=\left|\mathrm{E}_{\mathrm{e}}^{*}\right|+\frac{\left|\mathrm{E}_{\mathrm{g}}^{*}\right|-\left|\mathrm{E}_{\mathrm{e}}^{*}\right|}{\left[1+\left(\mathrm{fc} / \mathrm{f}^{\prime}\right)^{\mathrm{k}}\right]^{\mathrm{k} / \mathrm{m}} \mathrm{e}}
$$

onde: $\left|\mathrm{E}^{*}\right|$ - módulo complexo; $\left|\mathrm{E}_{\mathrm{e}}^{*}\right|-$ módulo complexo de equilíbrio, quando $f^{\prime} \rightarrow 0$; $\left|\mathrm{E}_{\mathrm{g}}^{*}\right|-$ módulo complexo de equilíbrio, quando $f^{\prime} \rightarrow \infty ; f_{c}$ - parâmetro de localização, com dimensões de frequência; $f^{\prime}$ - frequência reduzida; k, $\mathrm{m}_{\mathrm{e}}$ - parâmetros de forma, adimensionais.

Os parâmetros $\left|\mathrm{E}_{\mathrm{e}}^{*}\right| \mathrm{e}\left|\mathrm{E}_{\mathrm{g}}^{*}\right|$ podem ser obtidos diretamente com a prolongação gráfica da curva mestra, restando apenas determinar $f_{c}$, $\mathrm{k} \mathrm{e} \mathrm{m}_{\mathrm{e}}$ a partir do ajuste da função representada na Equação L. Caso não haja possibilidade de leitura dos extremos da curva mestra, todos os cinco parâmetros podem ser calculados pelo ajuste dos dados. Utilizou-se o software LAB Fit - Curve Fitting Software [19] para ajustar |E*|, dado pela Equação L, aos resultados experimentais, por meio da técnica da regressão múltipla não linear. Ressalta-se que o mencionado ajuste não se trata de um modelo de predição do módulo dinâmico, mas apenas uma forma de correlacionar os resultados por meio de uma equação, o que não implica em qualquer relação de causalidade. Nesse sentido, realizaramse inúmeros trabalhos na tentativa de se estabelecer uma relação causal entre as diversas variáveis envolvidas no problema, tais como os modelos baseados em analogias mecânicas simples (modelo generalizado de Burgers e a série de Prony), assim como os modelos fenomenológicos, a partir de ajustes de dados experimentais, o que tem sido bastante aceito, sobretudo diante da facilidade atual de métodos computacionais para tratamento das informações.

\section{RESULTADOS E DISCUSSÃO}

\section{Dosagem e caracterização mecânica da mistura}

A composição pela metodologia Superpave indica $62 \%$ de ASAC, $34 \%$ de areia MAO, e 4\% de cimento Portland para a dosagem mineral, bem como $10,9 \%$ atinente ao teor de projeto. Ressalta-se que este valor se mostra bem acima dos resultados normalmente determinados para as misturas asfálticas confeccionadas com os agregados tradicionais (brita, seixo), o que pode ser explicado pela contribuição da alta absorção do agregado cerâmico. Os corpos de prova, produzidos com essa dosagem, apresentaram os resultados do módulo de rigidez dinâmico e ângulo de fase, de acordo
Tabela I - Módulo de rigidez dinâmico (MPa) para as diversas temperaturas e frequências.

[Table I-Dynamic modulus (MPa) for different temperatures and frequencies.]

\begin{tabular}{ccccc}
\hline $\begin{array}{c}\text { Temperatura } \\
\left({ }^{\circ} \mathrm{C}\right)\end{array}$ & 1 & 3 & 10 & 20 \\
\hline 25 & 1302,0 & 1608,0 & 1953,4 & 1998,2 \\
30 & 1048,6 & 1312,6 & 1649,8 & 1776,6 \\
35 & 712,6 & 940,4 & 1211,6 & 1268,2 \\
40 & 399,6 & 555,4 & 751,8 & 763,4 \\
45 & 235,4 & 331,4 & 457,4 & 534,0 \\
50 & 173,6 & 241,8 & 328,6 & 384,6 \\
55 & 107,0 & 150,2 & 202,0 & 351,6 \\
\hline
\end{tabular}

Tabela II - Ângulos de fase (grau) referentes aos valores médios.

[Table II - Phase angles (degree) relative to the average values.]

\begin{tabular}{ccccc}
\hline $\begin{array}{c}\text { Temperatura } \\
\left({ }^{\circ} \mathrm{C}\right)\end{array}$ & 1 & 3 & 10 & 20 \\
\hline 25 & 19,3 & 17,3 & 15,2 & 14,7 \\
30 & 22,7 & 17,4 & 14,6 & 17,3 \\
35 & 26,3 & 24,5 & 22,5 & 27,2 \\
40 & 31,8 & 30,7 & 29,7 & 39,2 \\
45 & 34,9 & 35,2 & 36,4 & 58,9 \\
50 & 35,3 & 36,8 & 40,8 & 71,8 \\
55 & 36,1 & 39,7 & 48,8 & 84,0 \\
\hline
\end{tabular}

com diversas frequências e temperaturas, expostos nas Tabelas I e II, respectivamente.

Curvas mestras do módulo de rigidez dinâmico (módulo complexo)

De posse das curvas de módulo dinâmico versus frequências para as diversas temperaturas, calcularam-se os fatores de translação horizontal $\left(\mathrm{a}_{\mathrm{T}}\right)$, conforme a Equação $\mathrm{K}$, utilizados para construção da curva mestra. Na Tabela III e Fig. 3 encontram-se tais resultados, considerando-se como referência as frequências a $40^{\circ} \mathrm{C}$.

$\mathrm{Na}$ curva mestra retratada na Fig. 3, notam-se alguns pontos fora do traçado geral. Tais pontos são oriundos dos valores concernentes ao ensaio com aplicação de carga à frequência de $20 \mathrm{~Hz}$, cujo comportamento mostra padrão distinto relativo aos demais. Esse resultado reforça um indicativo inicial de que eles não podem ser utilizados dentro do mesmo padrão de referência dos demais pontos, posto que o equipamento não foi capaz de induzir o deslocamento programado para essa frequência. Visando uma melhor visualização dos resultados, traçou-se uma nova curva mestra, excluindo-se os valores relativos àquela frequência (Fig. 4).

A partir da curva mestra para $40^{\circ} \mathrm{C}$ obtiveram-se valores do módulo de rigidez com frequências entre 0,01 e $1200 \mathrm{~Hz}$, impossíveis de serem determinados diretamente nos ensaios 
Tabela III - Fatores de translação horizontal do módulo dinâmico, referente a $40{ }^{\circ} \mathrm{C}$.

[Table III - Shift factors of the dynamic modulus relative to $\left.40^{\circ} \mathrm{C}.\right]$

\begin{tabular}{|c|c|c|c|}
\hline $\begin{array}{c}\text { Temperatura, } \\
\mathrm{T}\left({ }^{\circ} \mathrm{C}\right)\end{array}$ & $\begin{array}{l}\text { Shift factor, } \\
\qquad \alpha_{\mathrm{T}}\end{array}$ & $\begin{array}{l}\text { Frequência } \\
\text { reduzida, } \hat{\omega} \\
(\mathrm{Hz})\end{array}$ & $\begin{array}{c}\text { Módulo } \\
\text { dinâmico, IE* } \\
(\mathrm{MPa})\end{array}$ \\
\hline \multirow{4}{*}{55} & \multirow{4}{*}{0,008} & 0,008 & 107,0 \\
\hline & & 0,024 & 150,2 \\
\hline & & 0,08 & 202,0 \\
\hline & & 0,16 & 351,6 \\
\hline \multirow{4}{*}{50} & \multirow{4}{*}{0,05} & 0,05 & 173,6 \\
\hline & & 0,15 & 241,8 \\
\hline & & 0,5 & 328,6 \\
\hline & & 1 & 384,6 \\
\hline \multirow{4}{*}{45} & \multirow{4}{*}{0,17} & 0,17 & 235,4 \\
\hline & & 0,51 & 331,4 \\
\hline & & 1,7 & 457,4 \\
\hline & & 3,4 & 534,0 \\
\hline \multirow{4}{*}{40} & \multirow{4}{*}{1} & 1 & 399,6 \\
\hline & & 3 & 555,4 \\
\hline & & 10 & 751,8 \\
\hline & & 20 & 763,4 \\
\hline \multirow{4}{*}{35} & \multirow{4}{*}{8} & 8 & 712,6 \\
\hline & & 24 & 940,4 \\
\hline & & 80 & 1211,6 \\
\hline & & 160 & 1268,2 \\
\hline \multirow{4}{*}{30} & \multirow{4}{*}{40} & 40 & 1048,6 \\
\hline & & 120 & 1312,6 \\
\hline & & 400 & 1649,8 \\
\hline & & 800 & 1776,6 \\
\hline \multirow{4}{*}{25} & \multirow{4}{*}{120} & 120 & 1302,0 \\
\hline & & 360 & 1608,0 \\
\hline & & 1200 & 1953,4 \\
\hline & & 2400 & 1998,2 \\
\hline
\end{tabular}

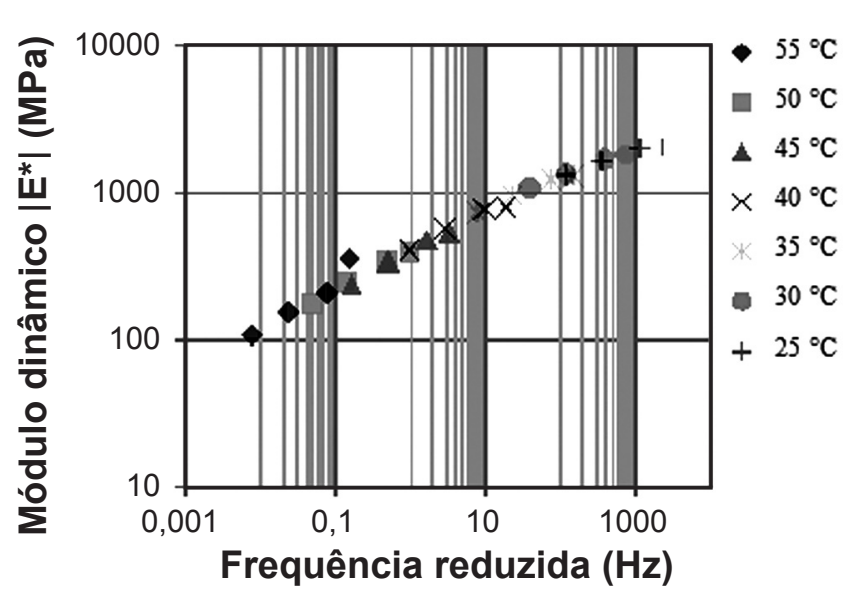

Figura 3: Curva mestra para o módulo dinâmico a $40{ }^{\circ} \mathrm{C}$. [Figure 3: Mastercurve for the dynamic modulus at $40^{\circ} \mathrm{C}$.]

laboratoriais. Igualmente, traçaram-se as curvas mestras para cada uma das temperaturas utilizadas nos testes. Para

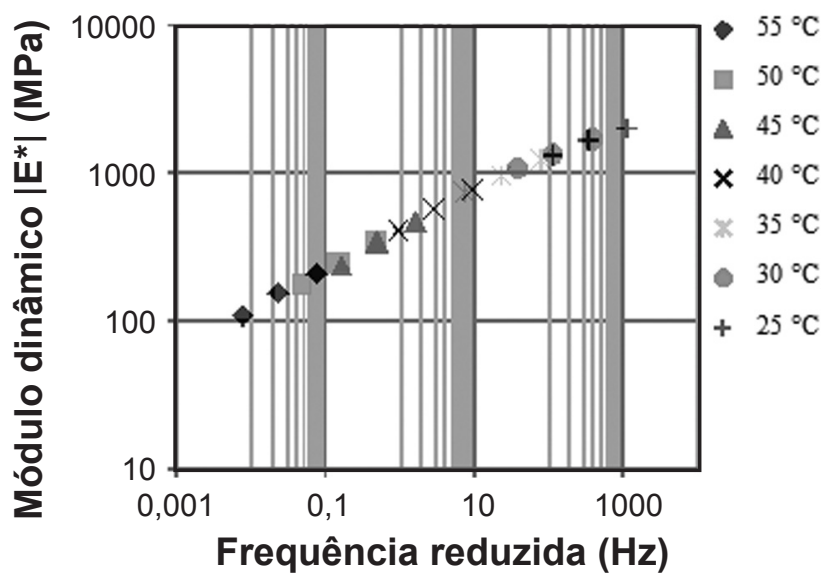

Figura 4: Curva mestra para o módulo dinâmico a $40{ }^{\circ} \mathrm{C}$, excluindose os resultados para $20 \mathrm{~Hz}$.

[Figure 4: Mastercurve for the dynamic modulus at $40{ }^{\circ} \mathrm{C}$, excluding the results for frequency of $20 \mathrm{~Hz}$.]

Tabela IV - Fatores de translação horizontal do módulo dinâmico, referente a $25^{\circ} \mathrm{C}$.

[Table IV - Shift factors of the dynamic modulus relative to $\left.25^{\circ} \mathrm{C}.\right]$

\begin{tabular}{|c|c|c|c|}
\hline $\begin{array}{c}\text { Temperatura, } \\
\mathrm{T}\left({ }^{\circ} \mathrm{C}\right)\end{array}$ & $\begin{array}{c}\text { Shift factor, } \\
\alpha_{\mathrm{T}}\end{array}$ & $\begin{array}{c}\text { Frequência } \\
\text { reduzida, } \hat{\omega} \\
(\mathrm{Hz})\end{array}$ & $\begin{array}{c}\text { Módulo } \\
\text { dinâmico, } \\
\text { |E*| (MPa) }\end{array}$ \\
\hline \multirow{3}{*}{55} & \multirow{3}{*}{0,0002} & 0,0002 & 107,0 \\
\hline & & 0,0006 & 150,2 \\
\hline & & 0,002 & 202,0 \\
\hline \multirow{3}{*}{50} & \multirow{3}{*}{0,0008} & 0,0008 & 173,6 \\
\hline & & 0,0024 & 241,8 \\
\hline & & 0,008 & 328,6 \\
\hline \multirow{3}{*}{45} & \multirow{3}{*}{0,002} & 0,002 & 235,4 \\
\hline & & 0,006 & 331,4 \\
\hline & & 0,02 & 457,4 \\
\hline \multirow{3}{*}{40} & \multirow{3}{*}{0,01} & 0,01 & 399,6 \\
\hline & & 0,03 & 555,4 \\
\hline & & 0,1 & 751,8 \\
\hline \multirow{3}{*}{35} & \multirow{3}{*}{0,08} & 0,08 & 712,6 \\
\hline & & 0,24 & 940,4 \\
\hline & & 0,8 & 1211,6 \\
\hline \multirow{3}{*}{30} & \multirow{3}{*}{0,35} & 0,35 & 1048,6 \\
\hline & & 1,05 & 1312,6 \\
\hline & & 3,5 & 1649,8 \\
\hline \multirow{3}{*}{25} & \multirow{3}{*}{1} & 1 & 1302,0 \\
\hline & & 3 & 1608,0 \\
\hline & & 10 & 1953,4 \\
\hline
\end{tabular}

ilustrar, calcularam-se os fatores de translação horizontal $\left(\mathrm{a}_{\mathrm{T}}\right)$ tendo como referência 25 e $55^{\circ} \mathrm{C}$ (Tabelas IV e V), gerando os gráficos expostos nas Figs. 5 e 6.

Por fim, agruparam-se as curvas mestras em um único gráfico presente na Fig. 7. Neste, pode-se determinar graficamente o módulo de rigidez para diversas temperaturas e frequências, muito além das usadas nos experimentos. 
Tabela V - Fatores de translação horizontal do módulo dinâmico, referente a $55^{\circ} \mathrm{C}$.

[Table $V$-Shift factors of the dynamic modulus relative to $\left.55^{\circ} \mathrm{C}.\right]$

\begin{tabular}{|c|c|c|c|}
\hline $\begin{array}{c}\text { Temperatura, } \mathrm{T} \\
\left({ }^{\circ} \mathrm{C}\right)\end{array}$ & $\begin{array}{l}\text { Shift factor } \\
\qquad \alpha_{\mathrm{T}}\end{array}$ & $\begin{array}{l}\text { Frequência } \\
\text { reduzida, } \hat{\omega} \\
\quad(\mathrm{Hz})\end{array}$ & $\begin{array}{c}\text { Módulo } \\
\text { dinâmico, IE* } \\
(\mathrm{MPa})\end{array}$ \\
\hline \multirow{3}{*}{55} & \multirow{3}{*}{1} & 1 & 107,0 \\
\hline & & 3 & 150,2 \\
\hline & & 10 & 202,0 \\
\hline \multirow{3}{*}{50} & \multirow{3}{*}{6} & 6 & 173,6 \\
\hline & & 18 & 241,8 \\
\hline & & 60 & 328,6 \\
\hline \multirow{3}{*}{45} & \multirow{3}{*}{18} & 18 & 235,4 \\
\hline & & 54 & 331,4 \\
\hline & & 180 & 457,4 \\
\hline \multirow{3}{*}{40} & \multirow{3}{*}{100} & 100 & 399,6 \\
\hline & & 300 & 555,4 \\
\hline & & 1000 & 751,8 \\
\hline \multirow{3}{*}{35} & \multirow{3}{*}{850} & 850 & 712,6 \\
\hline & & 2550 & 940,4 \\
\hline & & 8500 & 1211,6 \\
\hline \multirow{3}{*}{30} & \multirow{3}{*}{5000} & 5000 & 1048,6 \\
\hline & & 15000 & 1312,6 \\
\hline & & 50000 & 1649,8 \\
\hline \multirow{3}{*}{25} & \multirow{3}{*}{15000} & 15000 & 1302,0 \\
\hline & & 45000 & 1608,0 \\
\hline & & 150000 & 1953,4 \\
\hline
\end{tabular}

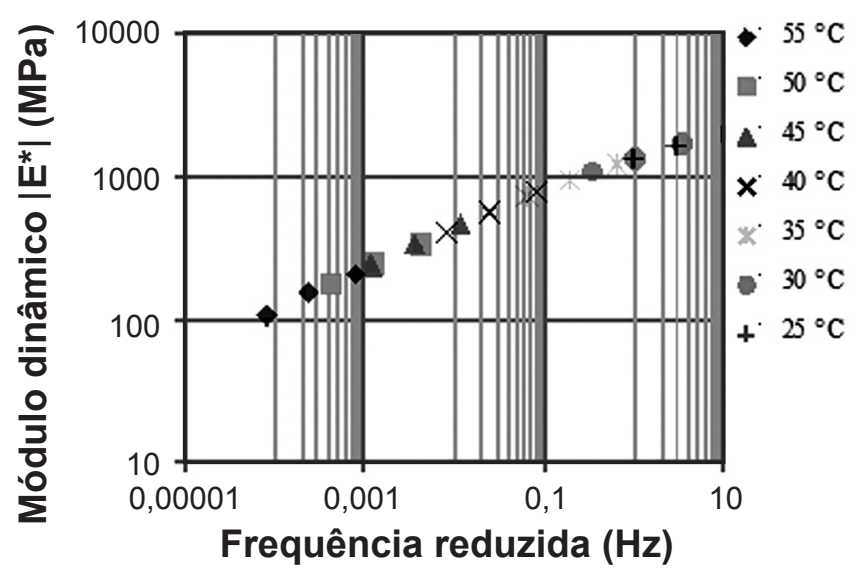

Figura 5: Curva mestra para o módulo dinâmico a $25^{\circ} \mathrm{C}$.

[Figure 5: Mastercurve for the dynamic modulus at $25^{\circ} \mathrm{C}$.]

Reside aí a grande importância das curvas mestras, que podem ampliar consideravelmente a fronteira dos dados. No caso específico, embora os ensaios válidos tenham se restringido a frequências compreendidas entre 1 e $10 \mathrm{~Hz}$, com a construção das curvas mestras, pode-se determinar o módulo para frequências entre 0,00002 e $100000 \mathrm{~Hz}$, a depender da temperatura. No caso das temperaturas intermediárias, podem-se interpolar outras curvas para obtenção dos resultados. Em suma, é possível encontrar

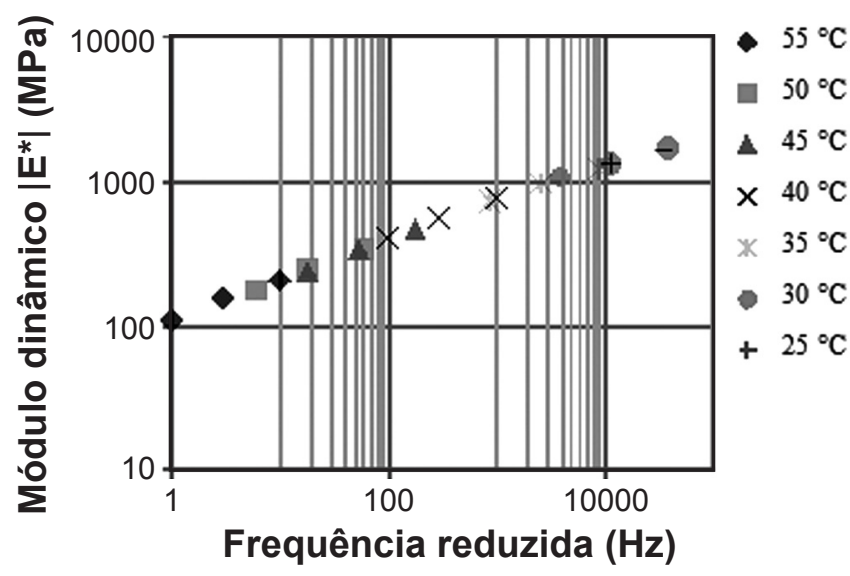

Figura 6: Curva mestra para o módulo dinâmico a $55^{\circ} \mathrm{C}$. [Figure 6: Mastercurve for the dynamic modulus at $55^{\circ} \mathrm{C}$.]

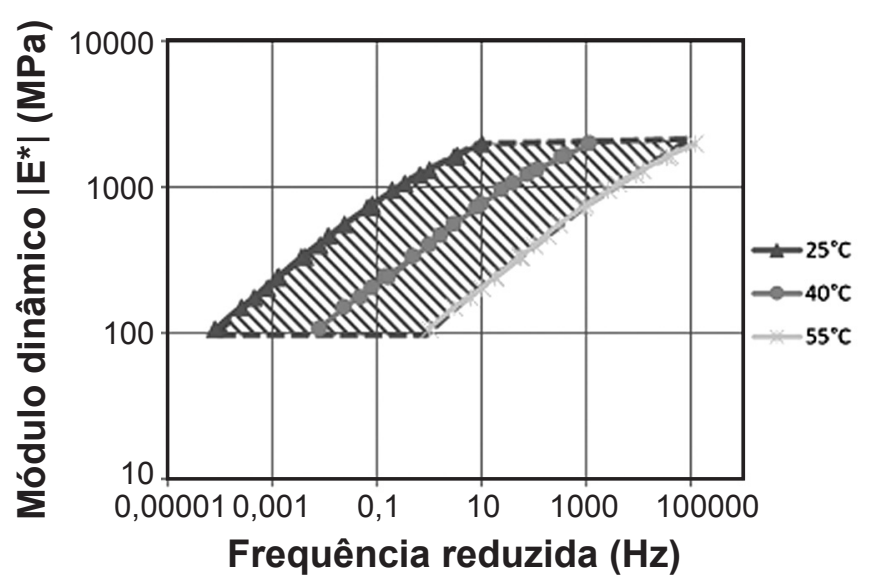

Figura 7: Curvas mestras para o módulo dinâmico em temperaturas entre 25 e $55^{\circ} \mathrm{C}$.

[Figure 7: Mastercurves for the dynamic modulus at temperatures between 25 and $55^{\circ} \mathrm{C}$.]

os valores do módulo dinâmico, por interpolação, para qualquer combinação de frequência e temperatura compreendida dentro da área abrangida pela medição, que na Fig. 7 está representada pele região hachurada, abrangida pela curva mestra de 25 a $55^{\circ} \mathrm{C}$.

Embora as curvas mestras tenham sido construídas com sucesso, o processo envolve trabalho considerável para cálculo dos fatores de translação horizontal. Também a necessidade de consulta gráfica, que objetiva um resultado para uma dada temperatura e frequência, impõe uma série de limitações à sua aplicação, sobretudo quanto à possibilidade do uso do resultado em métodos de análise analíticos ou numéricos. Para superar essas limitações, os resultados experimentais foram modelados com o uso do software do LAB Fit - Curve Fitting Software, sendo os dados de entrada mostrados na Tabela VI. Dentre as opções de equação apresentadas pelo software, selecionou-se a seguinte, com $\mathrm{R}^{2}=0,98: \mathrm{Y}=\mathrm{A} *(\mathrm{~B} * * \mathrm{X} 1) * \mathrm{X} 2 * * \mathrm{C}+\mathrm{D}$, com $\mathrm{A}=5670,166256 ; \mathrm{B}=0,954013843 ; \mathrm{C}=0,144204688$ e $\mathrm{D}=$ $-396,1123712$. Concisamente, representa-se da seguinte forma: 


$$
\left|E^{*}\right|=5670,16 \cdot 0,954^{\mathrm{T}} \cdot \omega^{0,1442}-396,11
$$

Para avaliação da qualidade dos dados provenientes da equação, selecionaram-se alguns pontos facilmente consultados no gráfico da Fig. 6 e compararam-se com os resultados obtidos pela Equação M (Tabela VII). A comparação demonstrou a eficiência e confiabilidade da

Tabela VI - Dados utilizados para regressão.

[Table VI - Data used for regression.]

\begin{tabular}{|c|c|c|}
\hline $\begin{array}{c}\text { Temperatura, } \mathrm{T} \\
\left({ }^{\circ} \mathrm{C}\right)\end{array}$ & $\begin{array}{l}\text { Frequência, } \omega \\
(\mathrm{Hz})\end{array}$ & $\begin{array}{l}\text { Módulo dinâmico, } \\
\qquad\left|\mathrm{E}^{*}\right|(\mathrm{MPa})\end{array}$ \\
\hline $\mathrm{X} 1$ & $\mathrm{X} 2$ & $Y$ \\
\hline 25 & 1 & 1302,0 \\
\hline 25 & 3 & 1608,0 \\
\hline 25 & 10 & 1953,4 \\
\hline 30 & 1 & 1048,6 \\
\hline 30 & 3 & 1312,6 \\
\hline 30 & 10 & 1649,8 \\
\hline 35 & 1 & 712,6 \\
\hline 35 & 3 & 940,4 \\
\hline 35 & 10 & 1211,6 \\
\hline 40 & 1 & 399,6 \\
\hline 40 & 3 & 555,4 \\
\hline 40 & 10 & 751,8 \\
\hline 45 & 1 & 235,4 \\
\hline 45 & 3 & 331,4 \\
\hline 45 & 10 & 457,4 \\
\hline 50 & 1 & 173,6 \\
\hline 50 & 3 & 241,8 \\
\hline 50 & 10 & 328,6 \\
\hline 55 & 1 & 107,0 \\
\hline 55 & 3 & 150,2 \\
\hline 55 & 10 & 202,0 \\
\hline
\end{tabular}

equação para determinação de valores dentro do intervalo de dados, apesar da divergência mais elevada que ocorreu para a frequência de $0,001 \mathrm{~Hz}$ e temperatura de $25^{\circ} \mathrm{C}$. Essa análise indica que todas as curvas mestras do material, representada pela região sombreada na Fig. 6, podem ser substituídas por uma equação obtida por meio de regressão múltipla não linear, com auxílio de ferramentas computacionais. A vantagem da regressão é que pode ser realizada de forma rápida, prática e eficiente, além de expressar o módulo dinâmico de forma contínua e permitir seu emprego em métodos analíticos ou numéricos, eventualmente utilizados para se estudar os pavimentos asfálticos.

Apesar do bom ajuste, a equação resultante da regressão não passa de simples correlações entre as variáveis e não expressa, a princípio, qualquer relação de causalidade, bem como não pode servir de parâmetro para qualquer tipo de extrapolação. Essa equação apenas substitui o trabalho de construção das curvas mestras e eventuais interpolações para determinação de valores intermediários. Por isso, seguiu-se com os trabalhos de ajustes, desta feita utilizando-se a Equação L. Realizou-se o ajuste para a curva mestra do módulo dinâmico apresentada na Fig. 5. Analisando-se graficamente pode-se extrair dessa curva, com prolongamento no sentido das menores frequências, $\mathrm{o}$ valor de $\left|\mathrm{E}_{\mathrm{e}}^{*}\right|=70 \mathrm{MPa}$. No entanto, não é possível encontrar o parâmetro $\left|\mathrm{E}_{\mathrm{g}}^{*}\right|$, correspondente ao módulo dinâmico para a frequência que tende ao infinito, diante da falta de obtenção do ponto de inflexão superior da curva mestra. Dessa forma, esse é mais um parâmetro $\left|\mathrm{E}_{\mathrm{g}}^{*}\right|$ a ser definido pelo processo de ajuste. Utilizando-se novamente o LAB Fit Curve Fitting Software, obteve-se, para a curva mestra referente a $40{ }^{\circ} \mathrm{C}$, um excelente ajuste, com $\mathrm{R}^{2}=0,999875$, representado pela seguinte equação:

$$
\left|E^{*}\right|=70+\frac{50003,62}{\left[1+\left(1,1753 / f^{\prime}\right)^{0,1783}\right]^{3,8280}}
$$

Da Equação N, de imediato extrai-se o módulo dinâmico para frequências que tendem ao infinito, indisponível até o momento, mesmo com a construção das

Tabela VII - Comparativo entre os resultados das curvas mestras e da Equação M resultante da regressão.

\begin{tabular}{|c|c|c|c|c|}
\hline $\begin{array}{c}\text { Temperatura, } \mathrm{T} \\
\left({ }^{\circ} \mathrm{C}\right)\end{array}$ & $\begin{array}{c}\text { Frequência, } \omega \\
(\mathrm{Hz})\end{array}$ & $\begin{array}{l}\left|\mathrm{E}^{*}\right| \text { obtido pelo } \\
\text { gráfico }(\mathrm{MPa})\end{array}$ & $\begin{array}{l}\left|\mathrm{E}^{*}\right| \text { obtido pela } \\
\text { equação }(\mathrm{MPa})\end{array}$ & Diferença $(\%)$ \\
\hline 25 & 0,001 & 200 & 249,3 & 24,7 \\
\hline 25 & 0,1 & 800 & 857,8 & 7,2 \\
\hline 25 & 10 & 1990 & 2039,8 & 2,5 \\
\hline 40 & 0,1 & 210 & 222,7 & 6,1 \\
\hline 40 & 10 & 790 & 806,1 & 2,0 \\
\hline 40 & 1000 & 1880 & 1939,5 & 3,2 \\
\hline 55 & 10 & 200 & 197,2 & $-1,4$ \\
\hline 55 & 1000 & 780 & 756,6 & $-3,0$ \\
\hline 55 & 100000 & 1900 & 1843,3 & $-3,0$ \\
\hline
\end{tabular}

[Table VII - Comparison between the results of mastercurves and the regression Equation M.] 


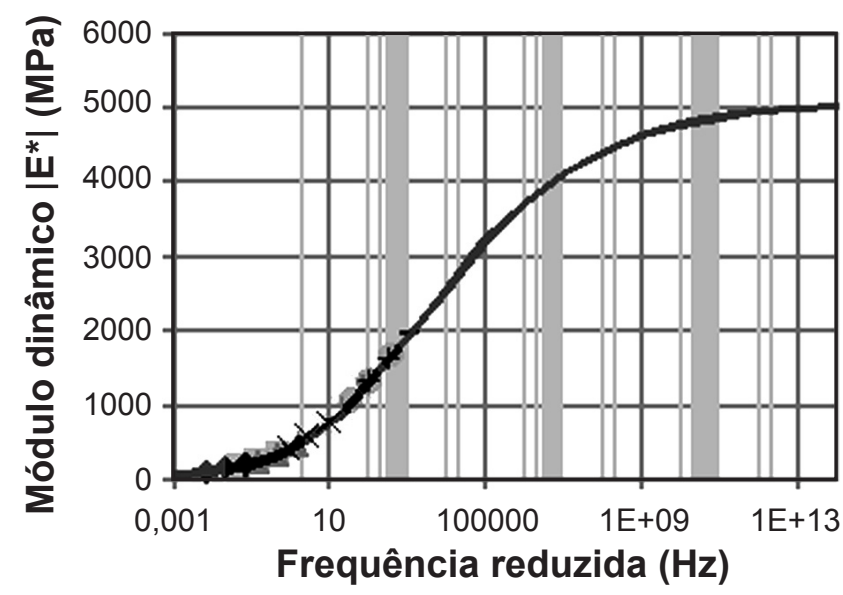

Figura 8: Curva representativa da equação de regressão do módulo dinâmico.

[Figure 8: Representative curve of the dynamic modulus regression function.]

curvas mestras. Comparando-se as Equações L e N, temse: $\quad\left|E_{\mathrm{g}}^{*}\right|-\left|E_{\mathrm{e}}^{*}\right|=5003,62 \rightarrow\left|E_{\mathrm{g}}^{*}\right|=4993,62 \quad$ Trata-se, por conseguinte, de um valor fora do conjunto de dados até aqui obtido, mas, diferente do ajuste aleatório realizado de início, a Equação L representa um comportamento universal das misturas asfálticas, razão pela qual se esperam valores coerentes para esse tipo de extrapolação. Na Fig. 8 tem-se a curva representativa da equação traçada sobre a curva mestra do módulo dinâmico, que se limitava à frequência de $1200 \mathrm{~Hz}$ e não definia o ponto de inflexão superior, comportamento esperado para o material em análise. Com a equação definida por Zeng et al. [18] pôde-se extrapolar os dados, passando-se pelo ponto de inflexão, a ponto de definir o patamar superior do módulo dinâmico, informação não obtida diretamente dos ensaios.

\section{CONCLUSÕES}

Para a mistura asfáltica com agregados sinterizados de argila calcinada (ASAC), sobrepondo-se as curvas de módulo dinâmico, $|\mathrm{E} *|$, versus frequência e aplicando-se os fatores de translação horizontal, obteve-se a curva mestra com excelente ajuste. Assim, para mistura asfáltica com ASAC, pôde-se obter o módulo dinâmico a frequências muito inferiores ou superiores às aplicadas diretamente nos ensaios laboratoriais, que são impossíveis de serem alcançadas experimentalmente em razão de limitação dos equipamentos. Pôde-se determinar uma curva mestra para cada temperatura ensaiada e o conjunto de curvas mestras representadas graficamente, e os valores obtidos diretamente dos testes podem ser ajustados com uso de recursos computacionais para serem retratados por uma função de duas variáveis, podendo-se, igualmente, calcular os valores de $\left|E^{*}\right|$ relativo a qualquer frequência e temperatura sem a realização de experimentos adicionais, desde que não haja extrapolações dos valores. Os modelos fenomenológicos representados pela Equação L podem ser aplicados para ajuste da curva mestra ao caso dos agregados sinterizados de argila calcinada. Obteve-se excelente coeficiente de correlação entre os valores da curva mestra para $40^{\circ} \mathrm{C}$, com $\mathrm{R}^{2}=0,999875$. Além de representar o módulo dinâmico de forma contínua, foi possível com essa equação extrapolar os dados, de onde se determinou o valor de equilíbrio do módulo dinâmico quando a frequência tende ao infinito.

\section{REFERÊNCIAS}

[1] C.A. Frota, F.R.G. Nunes, C.L. Silva, D.M. Melo, M.G.R. Santos, Cerâmica 53 (2007) 255-262.

[2] F.R.G. Nunes, C.L.C. Silva, C.A. Frota, Cerâmica 55 (2009) 425-436.

[3] A.C.L. Silva, C.A. Frota, Cerâmica 59 (2013) 508-517.

[4] A.C.L. Silva, C.A. Frota, Cerâmica 60 (2014) 10-21.

[5] R.L. Lytton, Transportation Research Record Nr. 1723, J. Transportation Res. Board 1723 (2000) 5-16.

[6] M.H. King, "Determination of Dynamic Moduli in Uniaxial Compression for North Caroline Hot Mix Asphalt Concrete", Master Thesis, North Carolina State University, Raleigh, NC (2004).

[7] ASTM - American Society for Testing and Materials C 136-95, "Standard Test Method for Sieve Analysis of Fine and Coarse Aggregates", USA (1995).

[8] ASTM - American Society for Testing and Materials, C 128-93, "Standard Test Method for Specific Gravity and Absorção of Fine Aggregate", USA (1988).

[9] AASHTO - American Association of State Highway and Transportation Officials, AASHTO T 19-93, "Standard Test Method for Unit Weight and Voids in Aggregate", USA (1997).

[10] SHRP - Superior Performing Asphalt Pavements (Superpave), "The Product of SHRP Asphalt Research Program", Superpave Series N. 1, Strategic Highway Research Program, Washington, DC (1994).

[11] SHRP - Superior Performance Asphalt Pavements (Superpave), "The Product of SHRP Asphalt Research Program", Superpave Series N. 2, Strategic Highway Research Program, Washington, DC (1994).

[12] T. Pellinen, B. Crockford, in $6^{\text {th }}$ International RILEM Symposium, Zurich (2003) 307-309.

[13] A.C.L. Silva, C.A. Frota, H.O. Frota, Matéria 20 (2015) 436-451.

[14] R.A. Schapery, Int. J. Fracture 25 (1984) 195-223.

[15] Y.R. Kim, Modeling of asphalt concret, McGraw-Hill eBook, USA (2009) 23.

[16] D.W. Christensen, D.A. Anderson, Proc. Assoc. Asphalt Pavement Technol. 61 (1992) 67-116.

[17] M.O. Marasteanu, D.A. Anderson, Eurobitume Workshop on Performance Related Properties for Bituminous Binders, Luxembourg (1999) 133.

[18] M. Zeng, H.U. Bahia, H. Zhai, M. Anderson, P. Turner, J. Assoc. Asphalt Paving Technol. 70 (2001) 403-441.

[19] W.P. Silva, C.M.D.P.S. Silva, "LAB Fit Curve Fitting Software", disponível em www.labfit.net, acesso em 21/12/2015.

(Rec. 23/06/2016, Ac. 30/06/2016) 